Original Research Paper

\title{
Harvesting Chaetoceros Calcitrans and Nannochloropsis sp. Via Electroflocculation
}

\author{
${ }^{1}$ Nitin Raut, ${ }^{2}$ Dinesh Kumar Saini and ${ }^{3}$ Reena Vaidya \\ ${ }^{1}$ Faculty of Engineering, Sohar University, Sohar, Oman \\ ${ }^{2}$ Faculty of Computing and IT, Sohar Universities, Sohar, Oman \\ ${ }^{3}$ Faculty of Science, Shree Shiivaji Science Colleges, Amravati, India
}

Article history

Received: 07-02-2016

Revised: 08-01-2017

Accepted: 30-03-2017

\section{Corresponding Author:}

Dinesh Kumar Saini

Faculty of Computing and

Information Technology, Sohar

University, Sohar, Oman

Email: dinesh@soharuni.edu.om

\begin{abstract}
The efficiency of harvesting the algal biomass plays a dominant role in the production of biodiesel from microalgae. On a commercial scale the choice and efficiency of the harvesting techniques may be a deciding factor for economic viability of this renewable energy source. The highly energy intensive centrifugation and filtration process are followed by flocculation for harvesting microalgae. Therefore the choice, cost and efficiency of the flocculating techniques can effectively lower the cost of harvesting and in turn minimize the cost of biodiesel production. This study aims to investigate the electroflocculation technique for harvesting microalgae under optimizing the operating parameters viz. voltage and current. Chaetoceros calcitrans and Nannochloropsis sp. were cultured and electrofloculated with a harvesting efficiency of 92 and $95 \%$ respectively at the optimum voltage and current.
\end{abstract}

Keywords: Electrodes, Efficiency, Electroflocculation, Harvesting, Microalgae, Optimum

\section{Introduction}

Microalgae (algae) are microscopic plants that are the primary synthesizers of organic matter in aquatic environment. They have high surface area-to-volume ratios, enabling the rapid uptake of nutrients and carbon dioxide $\left(\mathrm{CO}_{2}\right)$ and a much faster cell growth rate than land-based plant. Algae have been used in human food and health food product, feeds for fish larvae, shellfish and livestock and have been cultured for their high-value oils, chemicals, pharmaceutical product and pigments (Borowitzka and Borowitzka, 1990). Algae exists everywhere on the earth's surface and are primarily found in the soil and on the surface of the rocks, walls and tree trunks and over ice and polar ice and even in the dry deserts (Edzwald, 1995).

In the 1980 and $1990 \mathrm{~s}$, extensive research into the mass cultivation of algae biomass for the production of biofuels was conducted (Iverson, 2006). While the research showed this to be technically feasible, it also proved that, at the time, it was not economically feasible. The depressing results resulted in a less effort on microalgal research. However, recent concerns about increasing costs and decreasing first-generation biofuel feedstock's, has led to renewed interest and research into algae cultivation for bioenergy production (Balkan and Kolesnikova, 1996).
Electroflocculation is a technique that involves electrolytic addition of coagulating metal ions directly from sacrificial electrodes. These ions coagulate with pollutants in the water, in a similar manner to the addition of coagulating chemicals such as aluminium and iron chloride and allow the easier removal of the pollutants. The electrolytic addition of these ions has a number of advantages over their addition chemically. There is no addition of anions meaning no increase in salinity of the treated water. The system produces half to one third of the sludge (Borowitzka and Borowitzka, 1990).

Higher activity implies less metal ions requirement and a range of pollutants removal. In electroflocculation, the pollutants are removed by the bubbles which are generated during the process, capturing the coagulated pollutants and floating to the surface. Appropriately constructed, a single unit can be used to coagulate the pollutants and float them to the surface, from where they can be easily removed. Removal rates in excess of $98 \%$ have been achieved from a single stage process. Results from industrial installation confirm the theoretical benefits which can be obtained from this process.

\section{Literature Review}

The process of separation the microalgal mass from the culture medium termed as harvesting is one of the 
most difficult steps in the process of biodiesel production. Though, the harvesting step is the determinant to the economics of biodiesel production it is far less satisfactorily attended and resolved. The dry algal mass in the culture is comparatively very less and hence is difficult to achieve higher separation efficiencies and purity. There are a number of promising harvesting techniques but none of them are universally accepted as economic and efficient. The commercially explored harvesting techniques include centrifugation, filtration, sedimentation, flotation and flocculation (Levin et al., 1962).

The centrifugation techniques increases the speed of the particle by applying centrifugal force and the particle separates out based on density difference. This processes can be batch, semi batch or continuous and the centrifugation equipment is divided into fixed wall devices and rotating wall devices. The most commonly employed centrifuges for microalgal separation are discstack centrifuges, multi-chamber centrifuges, nozzle centrifuges and decanters (Levin et al., 1962). A disc stack centrifuge consists of a disc stack plates where the solids are removed continuously under gravitation and the clear liquid is collected from the top where as a multi-chamber centrifuge consists of vertical concentric cylinders. The feed is passed through these cylinders and is subjected to progressively higher accelerations. This results in deposition of larger particles at the inner chamber and finer particles at the outer chamber. A decanter centrifuge is very commonly used equipment for solid liquid separations which consists of a solid cylindrical bowl rotating at high speed inside which a screw conveyor is fixed rotating at slower speed, the solids are removed by the conveying motion and the clear liquid leaves the bowl at the other end.

Different filtration process can be employed to separate the microalgal mass; however the size of the microalgae and the filtration pressure limits its application (Lee et al., 2008). Operating filter presses to harvest microalgae are suitable only for large size of microalgae like Coelastrum proboscideum and Spirulina platensis but fail to recover organisms approaching bacterial dimensions, Scenedesmus, Dunaliella and Chlorella. However, using the diaphragm filter small size microalgae like Scenedesmus can be harvested with up to $100 \%$ filtration efficiency. Vacuum band filters are suitable for microalgae with more coarse particles like Spirulina but the cost required for operating these filters is very high and therefore these are not suitable for large scale filtration.

Sedimentation is a simple process of solid-liquid separation under gravity. There are two main typesclarification and thickening. The major importance in clarification is the clarity of the overflow where the feed suspension is usually dilute. In thickening the thickness of underflow is of more importance and the feed slurry is usually more concentrated (Svarovsky, 1979).

The advantages of sedimentation are low power requirement, low design cost and low manpower requirement. The sedimentation rates depend on cell density, cell size, cell motility and type of water flow (turbulent/laminar). In sedimentation the recoveries are too low for large scale harvesting however sedimentation can be used as one of the primary harvesting process to concentrate the microalgae (Geurts et al., 2009).

Flotation is a gravity separation process based on the attachment of air or gas bubbles to solid particles, which then are carried to the liquid surface and accumulate as float which can be skimmed off. The success of flotation depends on the instability of the suspended particles. The lower the instability the higher is the air particle contacts. The attachment of an air bubble to a particle depends on air, solid and aqueous phases contact angle. Smaller air bubbles are more efficient because of larger surface area per unit volume and lower buoyancy (Mishra and Jha, 2009). In this process air is continuously pumped into a flotation cell. Lower flotation efficiencies are due to the large size of the bubbles in froth flotation. The stability of the air-microalgae mixture can also be regulated by the $\mathrm{pH}$ of the culture. Not much literature is available on usage of froth flotation for large scale harvesting of microalgae, this process may not be suitable for harvesting microalgae due to lower flotation efficiencies (Mohn, 1980).

Dissolved air flotation is a separation process for the removal of solids by introducing a stream of fine air bubbles (Sina et al., 2012). Air supersaturated water is injected under pressure into a flotation cell. Higher floatation efficiencies are obtained due to micro-fine bubbles. Favourable flotation conditions for bubble attachment or adhesion to particles requires a reduction in the charge of particles, therefore, usually DAF is operated with chemical flocculants. Operating costs of DAF systems are usually higher than costs of sedimentation units due to higher cost requirements for producing micro-fine air bubbles and use of chemical flocculants (Moisan et al., 2002).

One of the most promising harvesting techniques of the microalgae produced appears to be flocculation. Microalgal cells carry a negative charge that prevents aggregation of cells in suspension. The surface charge can be neutralized or reduced by adding flocculants such as multivalent cations and cationic polymers to the broth. The flocculants used should ideally be inexpensive, nontoxic and effective in low concentration (El-Naas et al., 2009) and the selection of the flocculants should be so that further downstream processing is not adversely affected by its use. It is performed by adding specific chemicals to the algae water causing the algae to clump together. While this sounds easy and effortless, removing 
the chemical later may prove challenging and may add additional cost. Some examples of flocculants chemicals are chitosan or polyacrylamides. If some form of chemical flocculation is used, proper research must be undertaken to understand the effect of these chemical flocculants on the biomass contents (Suzuki and Takahash, 1995). Autoflocculation may be the best method. When the $\mathrm{CO}_{2}$ supply is interrupted (closed-system only) the algae will flocculate on its own (Becker, 1994). This seems at first thought to be great; however the efficiency of this method will not be high. Plus it would hardly work at all in an open-pond reactor if no $\mathrm{CO}_{2}$ was being bubbled through the water (Sukenik et al., 1988).

Electroflocculation is a common process for flocculating contaminants, organic matters and metal ions from waste water (El-Naas et al., 2009). Most of the literature available on electroflocculation technique is in the waste water treatment as well as hard water treatment (Chishti, 2007). The advantages of this method are that no addition of chemical flocculants is required and that similar cultivation conditions can be used for the flocculating microalgae as for the microalgae of interest that accumulate lipids (Chillie et al., 1966).

\section{Electroflocculation}

Electroflocculation is the process involving electrolytic addition of coagulating metal ions directly from sacrificial electrodes (Grima et al., 2003). The metal ions adsorbs the contaminants, ultrafine particles, oil drops and organic matters like algae, these coagulated precipitates then attach itself to the gas bubbles or are captured by the gas bubbles released during the process of electrolysis and floated to the top (Terry and Raymond, 1985).

The negative charge of the microalgae prevents aggregation of cells in suspension. In this method electrode dissolution and deposition takes place in the electrolyte culture solution, while the oxidation and reduction reaction take place on the surface of the electrodes. The anode plays a sacrificial electrode that donates positive metal ions into the solution. The positive ions then combine with the negatively charged microalgae to form (flocs) and these flocs eventually float to the top with the gas bubbles released at the cathodes and form a thin green mat that can be easily scrapped off. When the gas bubbles in the green mat escape the flocs settle to the bottom due to higher density (Azaria et al., 2007). To understand the process of electroflocculation it is really important to study the factors affecting the process of electroflocculation. The major factors controlling the process of electroflocculation are current, voltage, time, current density and electrode material, surface area of electrodes, $\mathrm{pH}$ and conductivity of the solution (Raut et al., 2015). Electroflocculation effectively removed the algal population from the reservoir water with a carry-over of $\mathrm{Al}^{3+}$ ions. Electroflocculation using different types of electrodes has been tested to harvest freshwater and marine algae resulting in overall flocculation efficiency higher than 90\% (Shinde et al., 2011).

\section{Current, Voltage and Time}

Current, voltage and time are three important factors that determine the power or cost required for electroflocculation. The power consumed by the electroflocculation unit is the product of voltage and current (Koren and Syversen, 1995). Amount of current passed through the culture solution will determine the ion concentration of the metal ions in the culture required to flocculate a certain volume of culture. At a fixed value of current the voltage will vary depending on the resistance offered by the culture solution. Lower the resistance offered by the culture solution less will be the voltage required (Zhu et al., 2005).

\section{Electrode Material}

The common material used in most electroflocculation processes is aluminum and better removal efficiencies were achieved using aluminium. However, not all of the references listed tried all the possible metal combinations. Use of iron $(\mathrm{Fe})$ rather than aluminum is also recommended as one of the better electrode materials due to fouling of aluminum and slower removal efficiencies, however these results were based on short term experiments (Balmer and Foulds, 1986). In the large scale harvesting of marine microalgae for biodiesel production, the electrode material plays an important role in the removal efficiency, cost, downstream treatment of the flocs, reuse of the supernatant and disposal of the final residual biomass. Therefore, taking these parameters into account the material chosen should be highly efficient in removal of microalgae, easily available and cheap. It must not interfere with the extraction process, safe for the growth of the microalgae; when the supernatant is reused and non-toxic to the environment when the residual biomass is disposed or able to be used as animal feed.

\section{Surface Area of Electrodes}

The surface area of electrodes will determine the size of electroflocculation setup. More the surface area of electrodes, higher will be the volume between the electrodes if the distance is kept constant and large volume of microalgae culture will be flocculated, although it will affect the current density and surface to volume ratio. Thus, for large scale electroflocculation setup, the surface area of electrodes could be determined by estimating the optimal surface to volume ratio and an array of bipolar electrodes are recommended for the flocculation of maximum volume of microalgae culture (Zenouzi et al., 2013). 


\section{Distance between Electrodes}

Distance is directly proportional to voltage, so less the distance between the electrodes, lower will be the required voltage to maintain the desirable amount of current flow/current density in the culture solution, however less volume of culture will be flocculated between the two electrodes.

Series of experiments needs to be performed to optimize the distance between electrodes for maximum culture flocculation at minimum power requirement. Shorter inter-electrode distances can cause short circuit due to high current density, therefore a distance of $2 \mathrm{~cm}$ should be preferred as the shortest distance.

\section{Materials and Methods}

The algal strains of Nannochloropsis sp. and Chaetoceros calcitrans as seen in Fig. 1a and 1b (Richmond and Zou, 1999) were cultivated in batch process under standard operating condition to produce a 25 lit volume of dense culture for electroflocculation.

Chaetoceros calcitrans and Nannochloropsis sp., both sea water microalgae were cultivated in sea water (Dodd, 1986). The ratio for microalgae cultivation was $70 \%$ water media and $30 \%$ cultured microalgae. The percentage of the fertilizer for a batch of one liter is 0.37 $\mathrm{mL}$ of the two fertilizers. The cultivation was done in a photo bioreactor with a light intensity of 1200 lux. Air was bubbled inside the reactor at a constant rate.
A two liter electroflocculator as in Fig. $2(15 \times 13 \times 10$ $\mathrm{cm})$ with two mesh electrodes $(12 \times 8 \mathrm{~cm})$ was designed. Electric current was supplied through a $12 \mathrm{~V}$ dc supply with a voltage regulator. A series of experiments were undertaken to study the effects of current, voltage and time on electro flocculation performance (Fig. 3a).

Initially a culture sample was subjected to low current (0.1-1.0A) to study the minimum current required for electro-flocculation. The current was then increased from 0.5 to $5.24 \mathrm{~A}$, in steps of $1 \mathrm{~A}$, to investigate the current tolerance limit of the microalgae and the time required for complete flocculation. A heavy duty D.C supply with a current limit of $5.24 \mathrm{~A}$ and a voltage limit of $18.5 \mathrm{~V}$ was used. Current and voltage values were noted from the ammeter and voltmeter, assembled in the D.C supply. A current of $0.1 \mathrm{~A}$ was observed to initiate electroflocculation; the electroflocculation rate was very slow the experiment was stopped after $1 \mathrm{~h}$, where only partial flocculation was achieved. Increasing the current decreased the flocculation time, which indicates that more current allows more ions to pass through the culture solution and time required for forming flocs, is reduced. A voltage of $16 \mathrm{~V}$ was required to achieve a current of $5 \mathrm{~A}$ and under this condition complete flocculation (complete flocculation here refers to removal efficiencies of 90$100 \%$ ) of a culture was achieved in $15 \mathrm{~min}$.

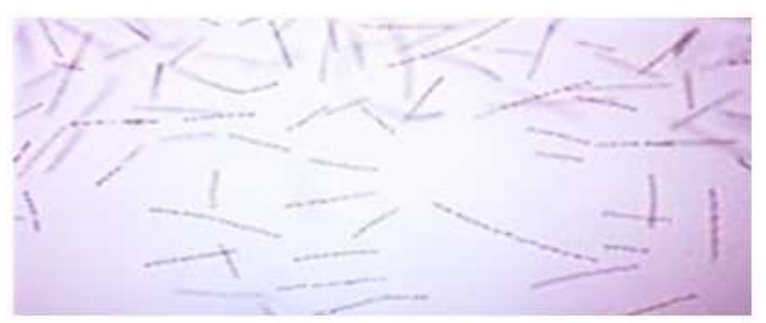

(b)

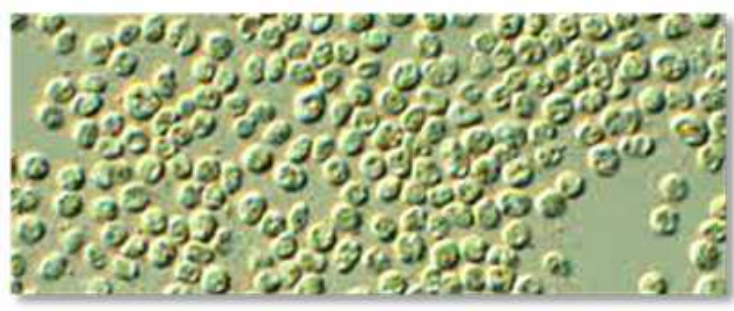

(a)

Fig. 1. (a) Nannochloropsis sp. (green microalgae), (b) Chaetoceros calcitrans (brown microalgae)

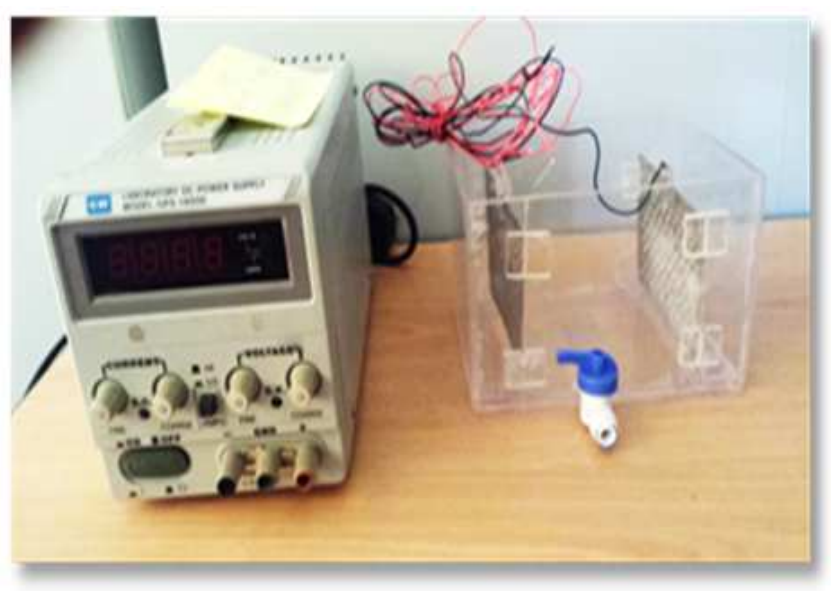

Fig. 2. Lab-scale electroflocculator 


\section{Results and Discussion}

Experiments were performed at lab-scale to apply the principle of electroflocculation to the Chaetoceros calcitrans and Nannochloropsis sp. microalgae species. Harvesting efficiency is the ratio of amount of biomass removal after electroflocculation to the initial biomass concentration in the culture solution:

$$
H E=\frac{V_{0} C_{0}-V_{f} C_{f}}{V_{0} C_{0}} \times 100 \%
$$

Where:

$V_{0}=$ Initial Volume of culture, $\mathrm{mL}$

$C_{0}=$ Initial Concentration of cells, $\mathrm{mg} / \mathrm{mL}$

$V_{f}=$ Volume of supernatant, $\mathrm{mL}$

$C_{f}=$ Concentration of cells in the supernatant, $\mathrm{mg} / \mathrm{mL}$

The harvesting efficiency of electroflocculation process was determined by counting the number of cells in the culture solution before and after flocculation using Equation 1.

Time plays an important role in determining the power required for complete flocculation of the microalgae without damaging the microalgae cells by charge flow. The power required can be calculated using Equation 2:

$$
P=V I t \times 100 \%
$$

Where:

$P=$ Power for electroflocculation, $\mathrm{kWh}$

$I=$ Current, amp

$V=$ Voltage, volts

$t=$ Time, sec

The experimental studies undertaken were aimed at arriving an optimum voltage and current, so as to achieve harvesting efficiency of $90 \%$ and above. Nannochloropsis

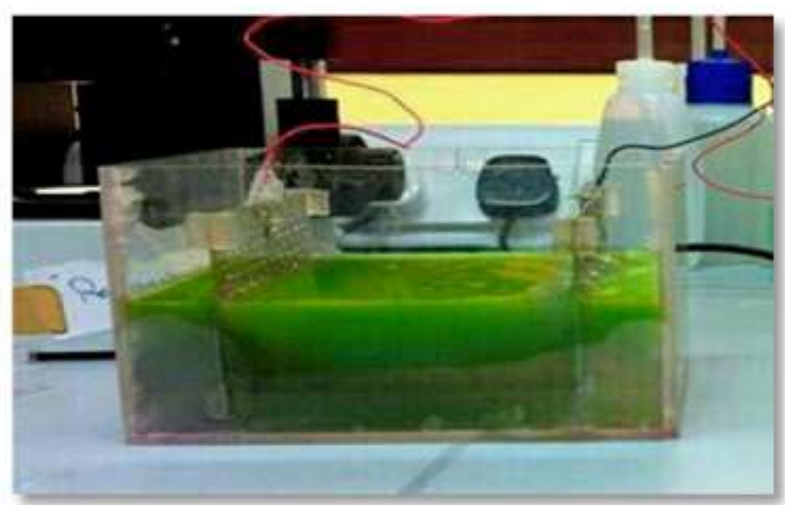

(a) sp. and Chaetoceros calcitrans of known density were fed to the electroflocculator and electroflucated as shown in Fig. $3 \mathrm{a}$ and $3 \mathrm{~b}$, at different current and voltages and the time to achieve harvesting efficiency of above $90 \%$ was noted. The current, voltage, time and electrode gap in the experimental studies were varied for both the microalgal species to estimate the harvesting efficiency and power consumed for electroflocculation.

\section{Effect of Voltage and Current on Harvesting Efficiency}

The effect of current and voltage on the HE for both microalgal species was investigated. As seen in Fig. 4, the electroflocculation time for Nannochloropsis sp. for a maximum HE of $90 \%$ was $380 \mathrm{sec}$. at a constant current of $5 \mathrm{~A}$, whereas for Chaetoceros calcitrans it was higher at $420 \mathrm{sec}$. The HE increased with the increase in voltage. These observations are in perfect agreement with earlier studies (Xu et al., 2010). Higher harvesting efficiencies can be achieved by operating at higher current, but an attempt to achieve harvesting efficiency beyond $90 \%$, at the outflow of current is not economically feasible. As observed in Fig. 5, 93\% HE was achieved for Nannochloropsis sp. within $480 \mathrm{sec}$, as against $88 \%$ for Chaetoceros calcitrans at a voltage of 15 V (Fig. 6 and 7), the HE varies gradually with time for Chaetoceros calcitrans as compared to the sharp increase for Nannochloropsis sp. The maximum HE of $94 \%$ was observed for both Nannochloropsis sp. and Chaetoceros calcitrans in $30 \mathrm{~min}$. at a voltage of $15 \mathrm{~V}$ and an alkaline $\mathrm{pH}$ of 10.5 which is in agreement with published data (Goldman et al., 1982).

\section{Effect of Current Density on Harvesting Efficiency}

The increase in current density definitely raises the harvesting efficiency, however the current density cannot be arbitrarily increased and an optimum current density needs to be estimated.

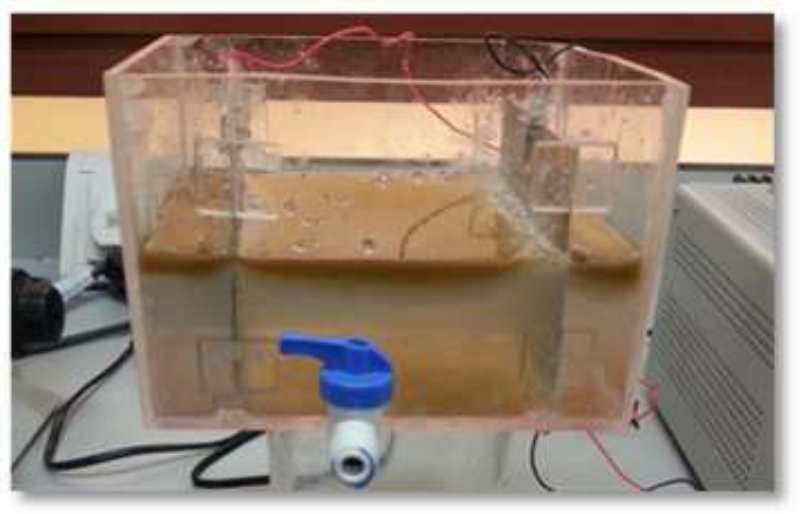

(b)

Fig. 3. (a) Electrofloculated Nannochloropsis sp., (b) Electrofloculated Chaetoceros calcitrans 


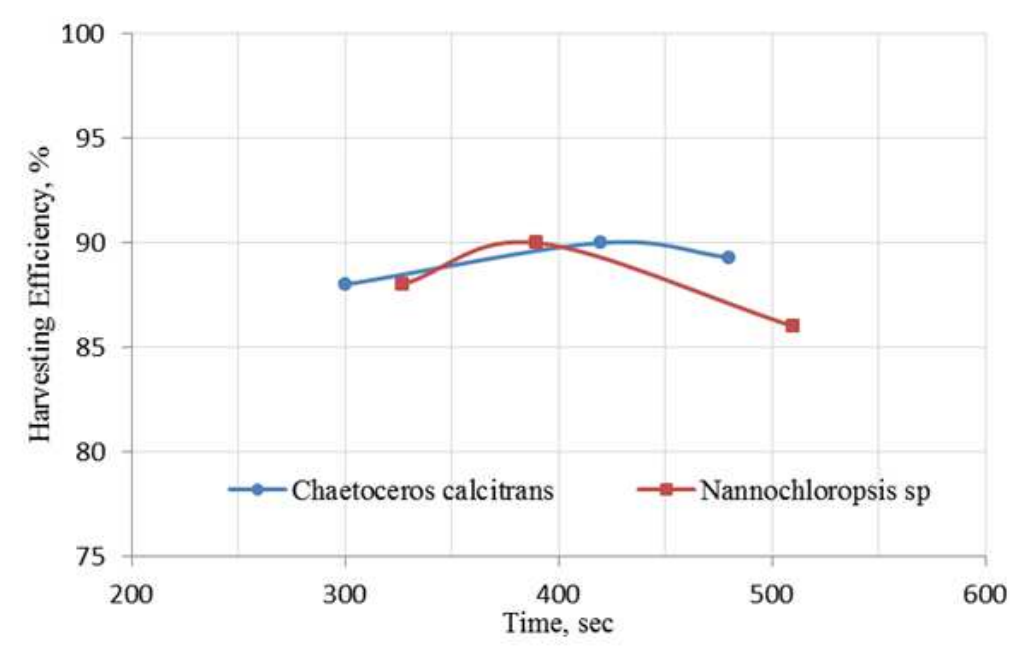

Fig. 4. Effect of flocculation time on $\mathrm{HE}$ at a constant I, $5 \mathrm{~A}$

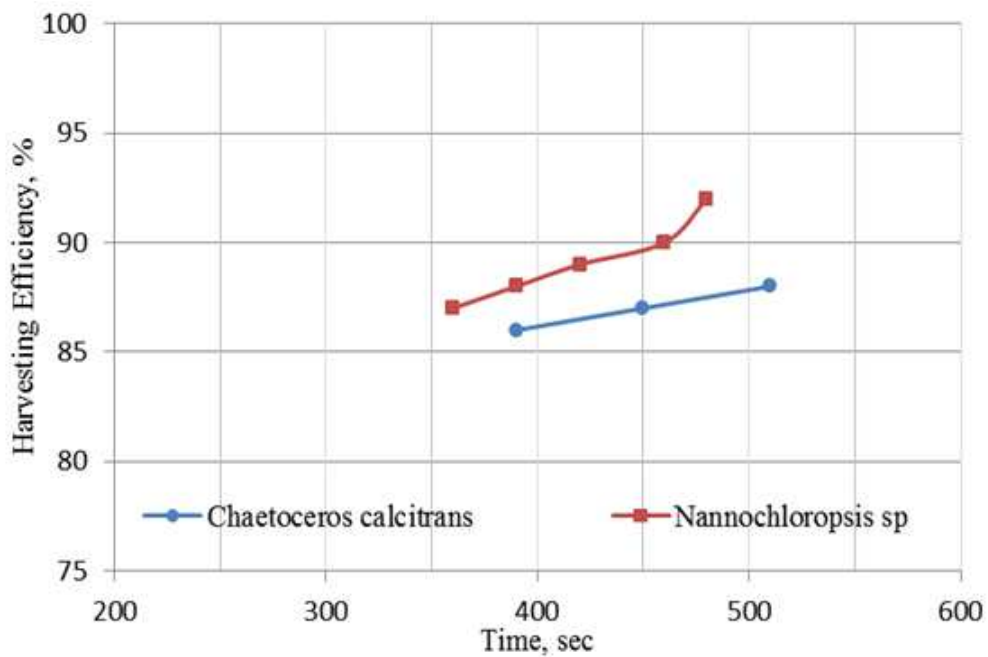

Fig. 5. Effect of flocculation time on $\mathrm{HE}$ at a constant V, $15 \mathrm{~V}$

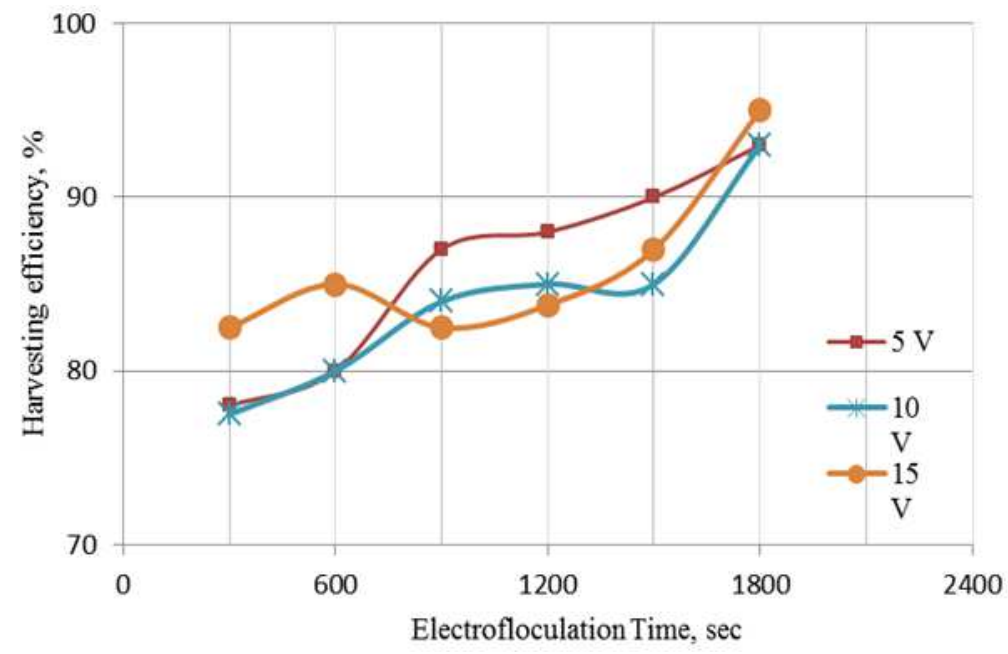

Fig. 6. Electroflocculation of nannochloropsis $\mathrm{sp} .(\mathrm{pH}=10.5$ varying voltages) 


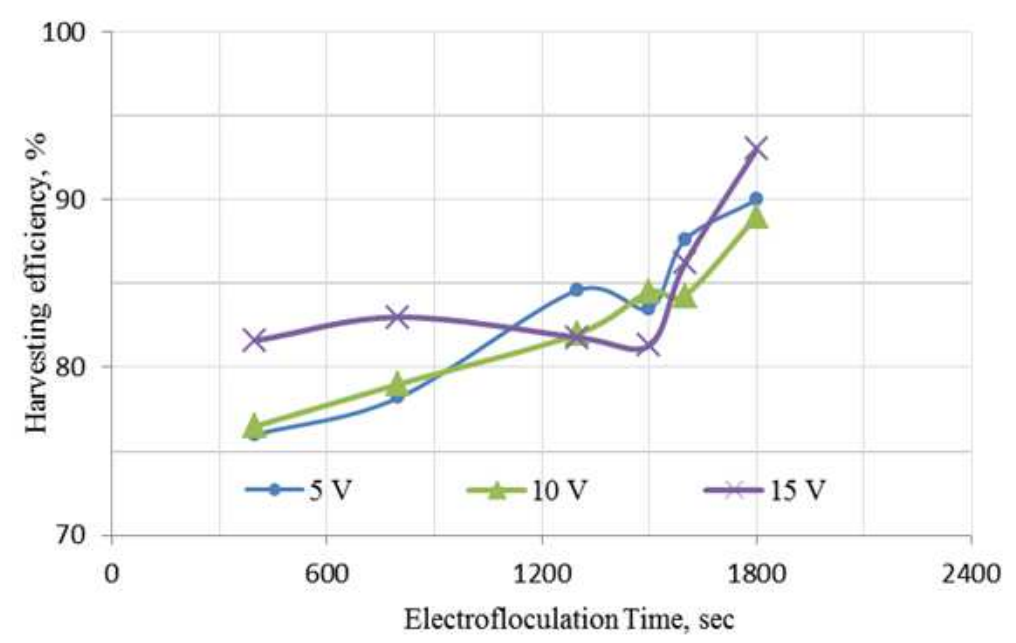

Fig. 7. Electroflocculation of chaetoceros calcitrans at $\mathrm{pH}=10.5$ and different voltages

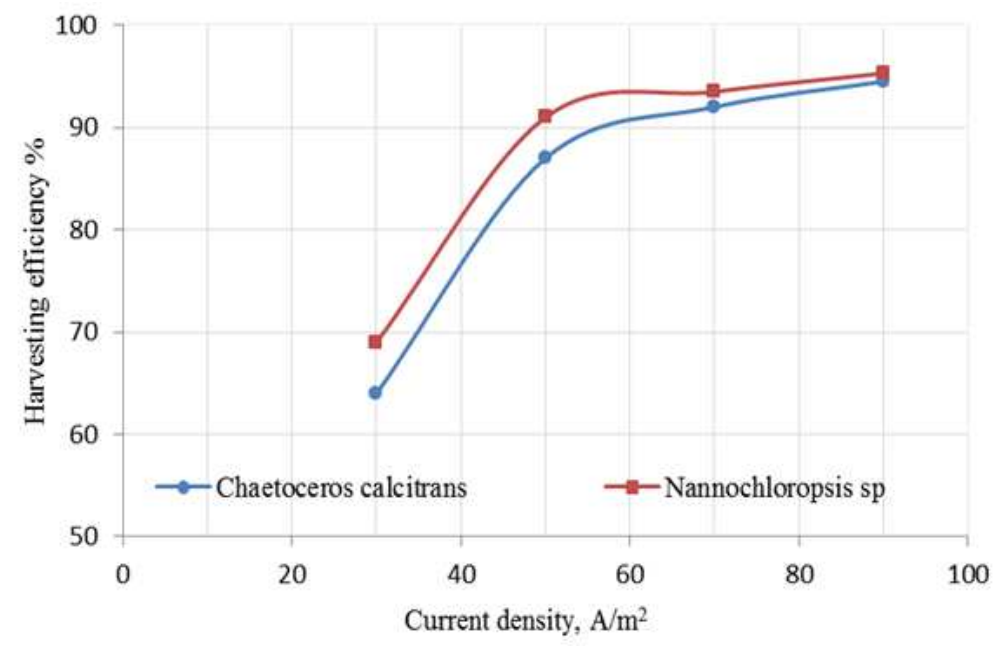

Fig. 8. Effect of current density on harvesting efficiency

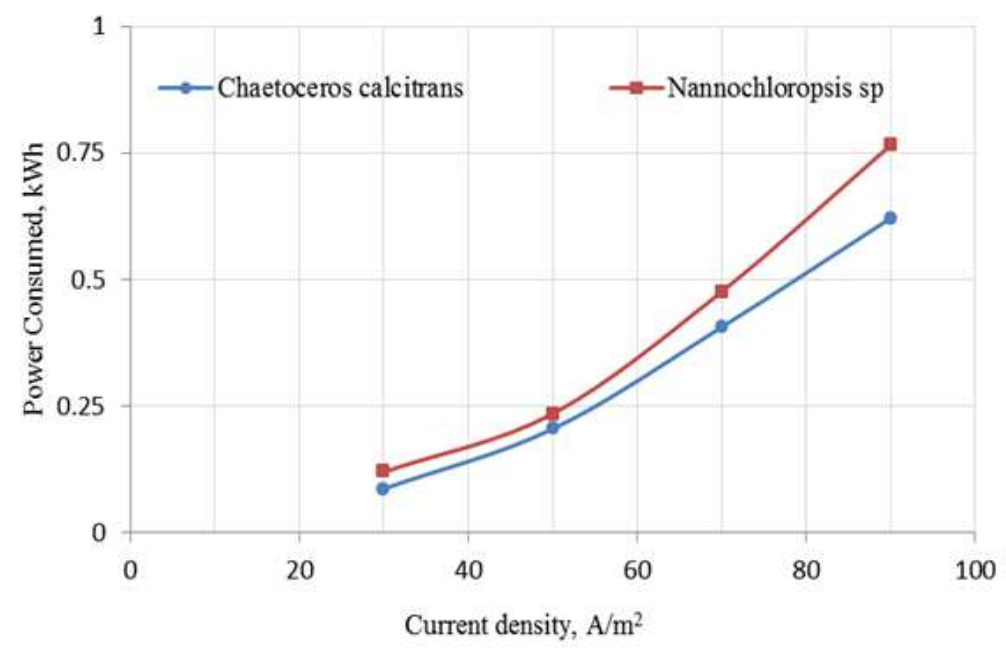

Fig. 9. Effect of current density on power consumption 


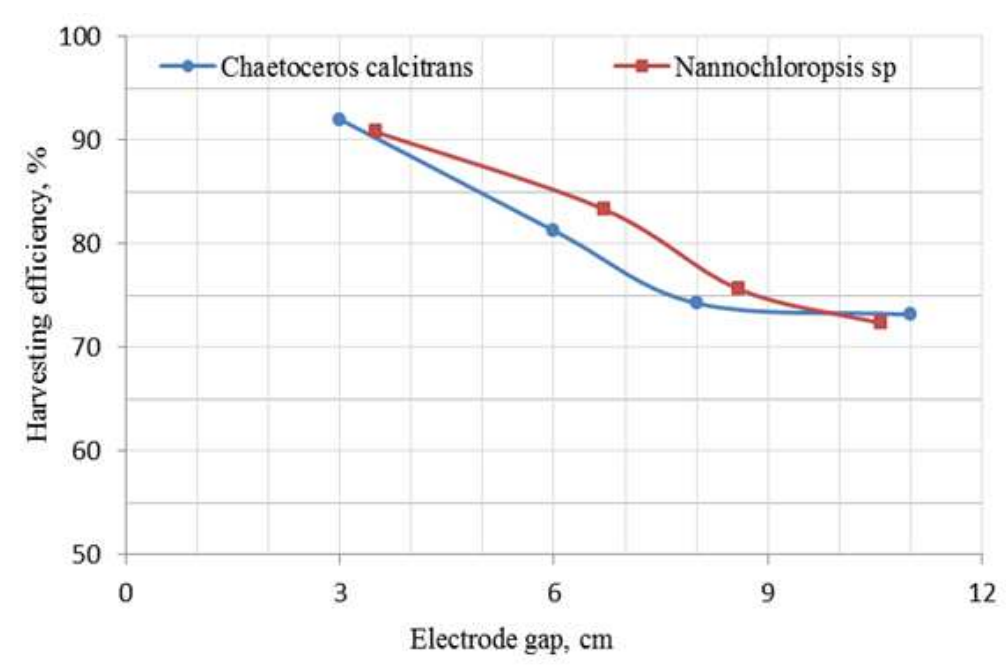

Fig. 10. Effect of electrode gap on HE

Figure 8 shows the effect of variation of current density on harvesting efficiency. For Chaetoceros calcitrans, the HE increased from 78 to $95 \%$ by increasing the current density from 40 to $80 \mathrm{~A} / \mathrm{m}^{2}$ whereas in case of Nannochloropsis sp., the HE reaches a maximum of 80 to $96 \%$. Higher $\mathrm{HE}$ at the expense of increased current density can be compromised. Because the power consumption efficiency increases for HE beyond $92 \%$ and also because the harvested algal biomass can be used as inoculum for fresh culturing of algae, as it was noticed that the optical density of unharvested Chaetoceros calcitrans and Nannochloropsis sp. increased on the third and fourth day respectively. The algal cell viability remains intact during the electroflocculation process.

\section{Effect of Current Density on Power Consumed}

It is but obvious that the attempt to achieve higher HE will result in higher power consumption making the process economically impractical. Figure 9 shows the effect of current density on the power consumption for both the microalgae species. At a current density of 90 $\mathrm{A} / \mathrm{m}^{2}$ the power consumed by Nannochloropsis $\mathrm{sp}$. is $0.76 \mathrm{kWh}$, as compared to $0.625 \mathrm{kWh}$ for Chaetoceros calcitrans. The difference in power consumption reduces at a lower current density.

\section{Effect of Electrode Gap on Harvesting Efficiency}

Experimental studies were conducted for studying the effect of the distance between the two electrodes on the harvesting efficiency and power consumed. It was observed for both the microalgal species that the power consumption for achieving a particular HE increased with increase in the electrode gap but a minimum electrode gap of $2 \mathrm{~cm}$ is recommended for electroflocculation. As observed from Fig. 10, for both, Nannochloropsis sp. and Chaetoceros calcitrans HE over
$90 \%$ can be achieved with an electrode gap of nearly 3 $\mathrm{cm}$, whereas the HE drops with increase in electrode gap.

\section{Conclusion}

Electroflocculation seems to be a feasible option for harvesting Nannochloropsis sp. and Chaetoceros calcitrans for achieving harvesting efficiencies over $90 \%$ and more. The electroflocculation time required for Nannochloropsis sp. is less as compared to Chaetoceros calcitrans under similar conditions. Also the harvesting efficiencies drops sharply for Nannochloropsis sp. as compared to the gradual drop in Chaetoceros calcitrans after reaching an optimum of $90 \%$ at $15 \mathrm{~V}$ and $10.5 \mathrm{pH}$. Higher harvesting efficiencies can be achieved in case of Nannochloropsis sp. but as optimum current density needs to be carefully estimated in order to make the process economically feasible. The power consumption for electroflocculation is comparatively higher in case of Nannochloropsis sp.

The effect of electrode gap on the harvesting efficiencies was nearly same in both the species with a minimum electrode gap of $2 \mathrm{~cm}$. In general electroflocculation is a viable option for harvesting microalgal species; however a careful selection of the current, voltage and electrode gap for individual species is required for minimizing cost.

\section{Acknowledgement}

This work is part of the research titled "Harnessing clean and green energy via integrated treatment of industrial and domestic waste water" (ORG/EI/10/2009) sponsored by The Research Council, Oman. The authors are indebted to The Research Council, Oman for funding this project and giving an opportunity to realize its successful completion. Special thanks of the entire team at 
The Research Council, Oman who helped and co-operated to administer the project for successful completion.

\section{Funding Information}

There is no funding available for this publication.

\section{Author's Contributions}

All authors equally contributed in this work.

\section{Ethics}

This article is original and contains unpublished material. The corresponding author confirms that all of the other authors have read and approved the manuscript and no ethical issues involved.

\section{References}

Azaria, G.H., A.R. Mesdaghiana, F. Vaezi, R. Nabizadeh and D. Nematollahi, 2007. Algae removal by electrocoagulation process, application for treatment of the effluent from an industrial wastewater treatment plant. J. Public Health, 36: 57-64.

Balkan, V.Y. and I.P. Kolesnikova, 1996. Influence of electrode material on the electrocoagulation. J. Aerosol Sci., 27: S209-S210. DOI: 10.1016/0021-8502(96)00177-2

Balmer, L.M. and A.W. Foulds, 1986. Electroflocculation/electroflotation for the removal of oil from oil-in-water emulsions. Filtrat. Separat., 23: 366-366.

Becker, E.W., 1994. Microalgae: Biotechnology and Microbiology. 1st Edn., Cambridge University Press, Cambridge, ISBN-10: 0521350204, pp: 293.

Borowitzka, L.J. and M.A. Borowitzka, 1990. Commercial production of Beta-carotene by Dunaliella salina in open ponds. Bull. Marine Sci., 47: 244-252.

Chillie, G.G., I.R.J. Vuuren, G.J. Stander and F.F. Kolbe, 1966. The reclamation sewage effluent for domestic use.

Chishti, Y., 2007. Biodiesel from microalgae. Biotechnol. Adv., 25: 294-306. DOI: $10.1016 /$ j.biotechadv.2007.02.001

Dodd, J.C., 1986. Elements of Pond Design and Construction. In: CRC Handbook of Microalgal Mass Culture. Richmond, A. (Ed.), Taylor and Francis Group, Boca Raton, ISBN-10: 0849332400, pp: 265-284.

Edzwald, J.A., 1995. Principles and applications of dissolved air flotation. Water Sci. Technol., 31: 1-23. DOI: 10.1016/0273-1223(95)00200-7

El-Naas, M.H., S. Al-Zuhair, A. Al-Lobaney and S. Makhlouf, 2009. Assessment of electrocoagulation for the treatment of petroleum refinery wastewater. J. Environ. Manage., 91: 180-185. DOI: $10.1016 /$ j.jenvman.2009.08.003
Goldman, J.C., Y. Azov, C.B. Riley and M.R. Dennett, 1982. The effect of $\mathrm{pH}$ in intensive microalgal cultures. I. Biomass regulation. J. Exp. Marine Biol. Ecol., 57: 1-13. DOI: 10.1016/0022-0981(82)90140-X

Grima, E.M., E.H. Belarbi, F.G. Fernandez, M.A. Acien and A. Robles et al., 2003. Recovery of microalgal biomass and metabolites: Process options and economics. Biotechnol. Adv., 20: 491-515. DOI: 10.1016/S0734-9750(02)00050-2

Iverson, T.M., 2006. Evolution and unique bioenergetic mechanisms in oxygenic photosynthesis. Curr. Opin. Chem. Biol., 10: 91-100. DOI: 10.1016/j.cbpa.2006.02.013

Geurts, A.M., G.J. Cost, Y. Freyvert, B. Zeitler and J.C. Miller et al., 2009. Knockout rats via embryo microinjection of zinc-finger nucleases. Science, 325: 433-433.

Koren, J.P.F. and U. Syversen, 1995. State-of-the-art electroflocculation. Filtrat. Separat., 32: 153-156. DOI: 10.1016/S0015-1882(97)84039-6

Lee, A.K., D.M. Lewis and P.J. Ashman, 2008. Microbial flocculation, a potentially low-cost harvesting technique for marine microalgae for the production of biodiesel. J. Applied Phycol., 21: 559-567. DOI: 10.1007/s10811-008-9391-8

Levin, G.V., J.R. Clendenning, A. Gibor and F.D. Bogar, 1962. Harvesting of algae by froth flotation. Applied Environ. Microbiol., 10: 169-175.

Mishra, A. and B. Jha, 2009. Isolation and characterization of extracellular polymeric substances from micro-algae Dunaliellasalina under salt stress. Bioresource Technol., 100: 3382-3386. DOI: 10.1016/j.biortech.2009.02.006

Mohn, F.H., 1980. Experiences and Strategies in the Harvesting of Biomass from Mass Cultures of Microalgae. In: Algae Biomass: Production and Use, Shelef. G. and C.J. Soeder (Eds.), Elsevier/NorthHolland Biomedical Press, Amsterdam, pp: 547-571.

Moisan, J.R., T.A. Moisan and M.R. Abbott, 2002. Modelling the effect of temperature on the maximum growth rates of phytoplankton populations. Ecol. Modell., 153: 197-215. DOI: 10.1016/S0304-3800(02)00008-X

Raut, N.B., T. Al-Balushi, S. Panwar, R.S. Vaidya and G.B. Shinde, 2015. Microalgal biofuel.

Richmond, A. and N. Zou, 1999. Efficient utilisation of high photon irradiance for mass production of photoautotrophic micro-organisms. J. Applied Phycol., 11: 123-127. DOI: 10.1023/A:1008033906840

Shinde, G.B., V.S. Sapkal, R.S. Sapkal and N.B. Raut, 2011. Transesterification by Reactive Distillation for Synthesis and Characterization of Biodiesel. In: Biodiesel-Feedstocks and Processing Technologies, Stoytcheva, M. (Ed.). 
Sukenik, A., D. Bilanovic and G. Shelef, 1988. Flocculation of microalgae in brackish and sea waters. Biomass, 15: 187-199. DOI: 10.1016/0144-4565(88)90084-4

Sina, S., B. Rouke, H.V. Marian and R.H. Wijffels, 2012. Harvesting of microalgae by bio-flocculation. J. Applied Phycol., 23: 849-855. DOI: $10.1007 / \mathrm{s} 10811-010-9591-\mathrm{x}$

Suzuki, Y. and M. Takahashi, 1995. Growth responses of several diatom species isolated from various environments to temperature. J. Phycol., 31: 880-888. DOI: $10.1111 / \mathrm{j} .0022-3646.1995 .00880 . \mathrm{x}$

Svarovsky, L., 1979. Advances in solid/liquid separation. II. Sedimentation, centrifugation and flotation.

Terry, K.L. and L.P. Raymond, 1985. System design for the autotrophic production of microalgae. Enzyme Microbial Technol., 7: 474-487.

DOI: 10.1016/0141-0229(85)90148-6
Xu, L., F. Wang, Z.U. Li, Z.M. Hu and C. Guo et al., 2010. Development of an efficient electroflocculation technology integrated with dispersed-air flotation for harvesting microalgae. J. Chem. Technol. Biotechnol., 85: 1504-1507. DOI: 10.1002/jctb.2457

Zenouzi, A., B. Ghobadian, M.A. Hejazi and P. Rahnemoon, 2013. Harvesting of microalgae dunaliella salina using electroflocculation, J. Agr. Sci. Tech., 15: 879-888.

Zhu, B., D.A. Clifford and S. Chellam, 2005. Comparison of electrocoagulation and chemical coagulation pretreatment for enhanced virus removal using microfiltration membranes. Water Res., 39: 3098-3108. DOI: $10.1016 /$ j.watres.2005.05.020 